\title{
Filme, Onlinevideos und Videospiele
}

\section{Filme}

AbStrakter Film (D 2013). R: Birgit Hein. P: ---.

EAU ARgENTÉE, SYriE AUTOPORTRAIT (F 2013). R: Ossama Mohammed/Wiam Simav

Bedirxan. P: Les Films d'ici/Protaction Film.

Für EINE HANDvoll Dollar (Originaltitel: Per un pugno di dollari) (I/S/D 1964). R:

Sergio Leone. P: Arrigo Colombo/Giorgio Papi.

FÜR EIN PAAR DOLLAR MEHR (Originaltitel: Per qualche dollaro in più) (I/S/D 1965).

R: Sergio Leone. P: Arturo González/Alberto Grimaldi.

Hungry Bitches (BRA 2007). R: Marco Antônio Fiorito (as Marco Villanova). P:

MFX Media.

KRIEGSBILDER (D 2006). R: Birgit Hein. P: ---.

SNUFF (USA/ARG 1976). R: Micheal Findlay/Horacio Frederiksson. P: Jack Bravman/Allan Shackleton.

The Time That Remains (UK/I/B/F 2009). R: Elia Suleiman. P: Hani Farsi/Michael

Gentile.

The Uprising (B/UK 2013). R: Peter Snowdon. P: Bruno Tracq.

ZWei glorreiche Halunken (Originaltitel: 1 buono, il brutto, il cattivo) (I/S/D/USA 1966). R: Sergio Leone. P: Alberto Grimaldi.

\section{Onlinevideos}

91177info (2010): Neda's death was faked but she was later murdered (WARNING

GRAPHIC USE OF TOMATO SAUCE). In: Youtube.com vom 05.04.2010. Im

Internet unter: https://www.youtube.com/watch?v=E18bWaQL-Ao\&t=11s\&bpc $\operatorname{tr}=1544212191$ [07.12.2018].

aavo foas (2014): James Foley FAKE Execution Video Analysis Reveals the Truth. In:

Youtube.com vom 20.08.2014. Im Internet unter: https://www.youtube.com/w atch?v=lcUL7YoYaXo [30.08.2018].

Agent John Extra (2012): Chainsaw Beheading: REACTION! In: Youtube.com vom 21.01.2012. Im Internet unter: https://www.youtube.com/watch?v=orYHEL8F DQY [30.08.2018]. 
al-Jazeera English (2011). Syrian protesters capture own death on camera. In: Youtube.com vom 04.07.2011. Im Internet unter: www.youtube.com/watch?v=QnqiQICRD8w [23.08.2016].

$\mathrm{AM}+(2015)$ : Staged ISIS Execution Video Leaked By Hackers. In: Youtube.com vom 13.07.2015. Im Internet unter: https://www.youtube.com/watch?v=05-z7ijK5sc [30.08.2018].

Anouli Patchouli (2012): Touching Reality, Thomas Hirschhorn. In: Vimeo.com vom 12.12.2012. Im Internet unter: https://vimeo.com/55482318 [31.10.2018].

beerech (2015): ISIS Bloopers. In: Youtube.com vom 02.02.2015. Im Internet unter: https://www.youtube.com/watch?v=Qz3oWmDUW1Q [30.08.2018].

BILD.DE (2016): Video des Attentäters - ISIS-Anschlag bei Würzburg. In: Youtube.com vom 20.07.2016. Im Internet unter: https://www.youtube.com/wat ch?v=OeN41YJFWl8 [02.09.2017].

Canadian Conspiracy (2014): How To Easily Stage A Fake ISIS Beheading (LIVE)! In: Youtube.com vom 30.09.2014. Im Internet unter: https:/www.youtube.co $\mathrm{m} /$ watch?v=Orhv5U8IFPw [30.08.2018].

CBS (2012): Cell phone videos show horrors inside Syria. In: Youtube.com vom 21.02.2012. Im Internet unter: https://www.youtube.com/watch?v=Ov9F8vfo_ WI [30.09.2018].

clipmedia (2012): documenta 13 - Rabih Mroué The Pixelated Revolution. In: Artort.tv vom 15.06.2012. Im Internet unter: www.artort.tv/allgemein/document a-13-rabih-mroue-the-pixelated-revolution/ [04.01.2019].

CNN (2013): Syrian rebel eats heart, threatens regime. In: Youtube.com vom 16.05.2013. Im Internet unter: https://www.youtube.com/watch?v=LEZUraRo $\mathrm{r} 10 \& \mathrm{t}=20$ s [30.09.2018].

Dario Ré (2016): Touching Reality, 2014. In: Vimeo.com vom 29.09.2016. Im Internet unter: https://vimeo.com/184926876 [31.10.2018].

Deek Jackson (2014): FILMED TO DEATH IN SYRIA, UKRAINE, EGYPT, IRAQ AFGHANISTAN ETC bla bla. In: Youtube.de vom 22.05.2014. Im Internet unter: https:/www.youtube.com/watch?v=h50Cm6IFIbw [06.09.2017].

detectivemoonk (2009): neda video is fake - iranian woman shot dead is fake. In: Youtube.com vom 29.06.2009. Im Internet unter: https://www.youtube.com/w atch?v=CByrgILwGaM\&bpctr=1530607563 [29.05.2018].

Era Clinton (2014): Documentary Films - Original No Beheading -James Wright Foley Video. In: Youtube.com 23.08.2014. Im Internet unter: https://www.yout ube.com/watch?v=0zRK6vVdEow\&t=42s [30.08.2018].

Ersterreicherr (2015): ISIS-Enthauptung mitten in WIEN | Mariahilfer Straße 21.12.2015. In: Youtube.com vom 21.12.2015. Im Internet unter: https://www. youtube.com/watch?v=WwRV2bYGa5E [30.08.2018]. 
EsToSa S (2013): Syrian Child cries at the death of his big brother "EMOTIONAL". In: Youtube.com vom 14.07.2013. Im Internet unter: https:/www.youtube.com /watch?v=QpogpTmVRBo [02.05.2018].

ewretr33 (2012): CNN Farewell to Basil Al Sayed 1230 11. In: Youtube.com vom 07.05.2012. Im Internet unter: https:/www.youtube.com/watch?v=OLB3aJYg $7 \mathrm{vY}$ [30.08.2017].

FEELTHELIGHT (2009): Iran, Tehran: wounded girl dying in front of camera, Her name was Neda. In: Youtube.com vom 20.06.2009. Im Internet unter: www.y outube.com/watch?v=bbdEfoQRsLM [07.12.2018].

firas tlass (2013): Syria, Al Nabk, +18 Leaked video, Assad's thugs torture, abuse and murder civilians heinously. In: Youtube.com vom 07.03.2013. Im Internet unter: https://www.youtube.com/watch?v=swnm86F-3Wc [29.09.2018].

ForMotherSyria (2013): +21 Al Qaeda< rebel in Syria, rips out the heart of a Syrian and eats it. In: Youtube.com vom 06.09.2013. Im Internet unter: https://www. youtube.com/watch?v=q-rPdwloarc [30.09.2018].

GazpromFan18 (2013a): 18+not for shock!) Headshot! Syrian sniper kills a terrorist of CIA FSA (LIVE). In: Youtube.com vom 19.06.2013. Im Internet unter: https:/ /www.youtube.com/watch?v=wBtChitoW34 [02.05.2018].

GazpromFan18 (2013b): 18+ not for shock! just for fun ; Syria FSA attack on SAA outpost goes wrong. In: Youtube.com vom 19.06.2013. Im Internet unter: https ://www.youtube.com/watch?v=CmOCtbhtotw [02.05.2018].

hoyartiı (2011): Homs- Basel Sayyed's last camera footage before his death by Assad's thugs.22-12-2011. In: Youtube.com vom 30.12.2011. Im Internet unter: htt ps://www.youtube.com/watch?v=uYW3Ec_6rGI [03.01.2016].

I Am Kawehi (2013): Neda by Kawehi (WARNING: MATURE CONTENT) (Original by Kawehi). In: YouTube.com vom 16.01.2013. Im Internet unter: https://www. youtube.com/watch?v=k66S471J3as [07.012.2018].

imanemoprincess (2010): Nick Berg Beheading video - Chloe and Jo's Reaction. In: Youtube.com vom 16.04.2010. Im Internet unter: https://www.youtube.com/w atch?v=q-pi6o2Ryio [12.08.2018].

Islamska Predavanja (2013): Egyptian Photographer Films His Own Death - 18+ Army Sniper Shot Him-. In: Youtube.com vom 11.07.2013. Im Internet unter: h ttps://www.youtube.com/watch?v=Qest_tk7XFA [05.01.2019].

Jaime Mitropoulos (2009): NICK BERG DECAPITATION REACTION. In: Youtube.com vom 04.09.2009. Im Internet unter: https://www.youtube.com/wat ch?v=Cdgk79HHy_w [12.08.2018].

KARNEVAL MEGASTORE (2012): 2 Girls I Cup - Die 5 besten Reaktionen zum Ekelvideo. In: Youtube.com vom 30.11.2012. Im Internet unter: https://www.youtu be.com/watch?v=55rC4jMd_zA [30.08.2018]. 
Kiddam And The People (2016): KIDDAM AND THE PEOPLE \& 4dB »Neda« \#katp . In: Youtube.com vom 06.12.2016. Im Internet unter: https://www.youtube.co $\mathrm{m} /$ watch?v=3mNE9LWicN4 [07.12.2018].

Livegore.com (2017): Nicholas Berg Beheading. In: Livegore.com vom 16.12.2017. Im Internet unter: https://www.livegore.com/863/nicholas-berg-beheading [02.05.2018].

livingonplanetZ (2014): How They FAKED the James Foley Execution - SFX - Full Version. In: Liveleak.com vom 25.08.2014. Im Internet unter: https://www.live leak.com/view?t=85f_1409023450 [30.08.2018].

LV Art Studio (2014): TELETUBBIES GET KILLED - DIE CARTOON ANIMATION FAKE ISIS BEHEADING EXECUTION. In: Youtube.com vom 17.10.2014. Im Internet unter: https://www.youtube.com/watch?v=vxnVW36OgsU [30.08.2018].

Malarich (2016): Das Islam-Bekenntnis des Berlin-Terroristen Anis Amri. In: Youtube.com vom 24.12.2016. Im Internet unter: https://www.youtube.com/watch ?v=y50AMfPrDa4 [02.09.2017].

Mohamend Alahmed (2012): Syria: Homs: leaked video showing torturing of the civilian corpses 08 April 2012. In: Youtube.com vom 03.07.2012. Im Internet unter: https://www.youtube.com/watch?v=BKsXunwzGpU [29.09.2018].

MrSmith305 (2015): WTF: English Isis Music Video?!?!?!? In: Liveleak.com vom 02.12.2015. Im Internet unter: https://www.liveleak.com/view?t=db2_1449087 213 [30.08.2018].

MykeTysonFanio11 (2010): Photographer Leonardo Henrichsen filming his death. In: You-tube.com vom 26.10.2010. Im Internet unter: https:/www.youtube.co $\mathrm{m} /$ watch? $\mathrm{v}=\mathrm{lkVDHtSIfOk}$ [04.09.2017].

NaserSadr22 (2013): 18+not for shock! One FSA terrorist killed, another wounded by shrapnel shells in Idlib. In: Youtube.com vom 11.03.2013. Im Internet unter: https://www.youtube.com/watch?v=blZBkGNJH_o [03.08.2018].

netspanner (2011): Man films his own death in Syria protest. In: Youtube.com vom 04.07.2011. Im Internet unter: www.youtube.com/watch?v=j5JPFHL5rGk\&bpc $\operatorname{tr}=1369676086$ [23.08.2016].

NikiRyRyRyderx26 (2009): russian neo-nazi beheading reaction. In: Youtube.com vom 09.02.2009. Im Internet unter: https://www.youtube.com/watch?v=yVER UWuh5bI [30.08.2018].

Rebecca's News (2014): Horrifying Video. James Foley beheading, ISIS Terrorists Behead American Journalist. In: Youtube.com vom 19.08.2014. Im Internet unter: https://www.youtube.com/watch?v=GOyaJNKTj2w\&t=20s\&bpctr=1548060 852 [30.08.2018].

RPGtime (2018a): Path Out, pt: 1. In: Youtube.com vom 31.05.2018. Im Internet unter: https://www.youtube.com/watch? $\mathrm{v}=\mathrm{Og}$ - $7 \mathrm{Lxs} 8 \mathrm{X}$ o\&index=1\&list=PL9AZ Ioe2SWnNIbncX_S3yU_RyoRKh5DA5 [23.11.2018]. 
RPGtime (2018b): Path Out, pt:2. In: Youtube.com vom 04.06.2018. Im Internet unter: https:/www.youtube.com/watch?v=blUXiig4j1E\&list=PL9AZIoe2SWnNIb ncX_S3yU_RyoRKh5DA5\&index=2 [23.11.2018].

RPGtime (2018c): Path Out, pt:3. In: Youtube.com vom 05.06.2018. Im Internet unter: https://www.youtube.com/watch?v=-jwCgrWlGVE\&list=PL9AZIoe2SWnN IbncX_S3yU_RyoRKh5DA5\&index=3 [23.11.2018].

RPGtime (2018d): Path Out: Finale. In: Youtube.com vom 07.06.2018. Im Internet unter: https://www.youtube.com/watch?v=gazbOC70C4s\&list=PL9AZIoe2 SWnNIbncX_S3yU_RyoRKh5DA5\&index=4 [23.11.2018].

R\&U Videos (2018): GoPro Cam video taken off a dead ISIS jihadi | December 2018

| Syria (full version). In: Youtube.com vom 27.12.2018. Im Internet unter: http s://www.youtube.com/watch?v=Wjh5iKJ16Lg [04.01.2019].

SaraNews (2015): 18 Syrian sniper killed precise shot to the head of the terrorist FSA. In: Youtube.com vom 06.04.2015. Im Internet unter: https://www.youtub e.com/watch?v=Jcun_mVXyLE [02.05.2018].

SNN Shaam English News (2013a): SNN | Syria | Damascus Rural | Soldier's Grief Over Dead Brother | Jun 17, 2013. In: Youtube.com vom 17.06.2013. Im Internet unter: https://www.youtube.com/watch?v=H-PyP6QXgiQ [02.05.2018].

SNN Shaam English News (2013b): SNN | Syria | Dar'aa | Father Refuses To Believe Son Is Dead | Oct 8, 2013 | 18+ ONLY. In: Youtube.com vom 08.10.2013. Im Internet unter: https://www.youtube.com/watch?v=tPvFPE9C9sI\&bpctr=15315 64588 [02.05.2018].

souriamyheart (2012a): Assad Shabiha Cell Phone Secrets pt2 12812 Hama. In: Youtube.com vom 23.04.2012. Im Internet unter: https://www.youtube.com/w atch?v=MMM_8hBhLww [28.09.2018].

souriamyheart (2012b): Shabiha Cell Phone Secrets Self Video of Homs Massacre 1 29 12. In: Youtube.com vom 27.04.2012. Im Internet unter: https://www.youtu be.com/watch?v=rQuosPLk23k [28.09.2018].

squarerootofjess (2016): I'd Rather Be Alive - SEDA 12. In: Youtube.com 12.04.2016. Im Internet unter: https://www.youtube.com/watch?v=htvGYi93C D8 [07.12.2018].

squidd81 (2016): ISIS Insurgent films own death on GoPro - Graphic Footage. In: Youtube.com vom 10.09.2016. Im Internet unter: https://www.youtube.com/w atch?v=atRahVlineo [29.05.2018].

SyrRevoDiary English (2013): Leaked video showing Shabiha playing with the corpse of a Free Syrian Army soilder. 18+. In:Youtube.com vom 09.02.2013. Im Internet unter: https://www.youtube.com/watch?v=oovGmF2qSkQ [28.09.2018].

The Telegraph (2011): Cameraman in Yemen films his own shooting. In: Youtube.com vom 19.09.2011. Im Internet unter: https://www.youtube.com/watc $h ? v=4 N 6$ sICxgSI [05.01.2019]. 
TriLingua Daniyar NAURIZ (2014): FULL UNCENSORED VIDEO OF THE JAMES FOLEY BEHEADING. In: blog.daniyar.info vom 20.08.2014. Im Internet unter: https://blog.daniyar.info/2014/08/full-uncensored-video-of-the-james-fol ey-beheading/ [16.09.2017].

TomoNews US (2013): Egypt protest 2013: Video journalist films own death from sniper shot. In: Youtube.com vom 10.07.2013. Im Internet unter: https://www. youtube.com/watch?v=r-L2ZS6Qcwg [05.01.2019].

Walker Art Center (2012): Rabih Mroué in Conversation with Philip Bither. In: Youtube.com vom 01.02.2012. Im Internet unter: https://www.youtube.com/watc h?v=ZYXxPIh7zPo [04.01.2019].

WarLeaks (2014): Syria Civil War - Clashes And Insane Heavy Intense Urban Combat Firefight Helmet Cam Fighting Action. In: Youtube.com vom 23.03.2014. Im Internet unter https:/www.youtube.com/watch?v=tfEjTgUme Ww [04.01.2019].

WRO Art Center (2013): RABIH MROUÉ - THE PIXELATED REVOLUTION - documentation of artwork at Biennale WRO 2013. In: Vimeo.com vom 12.02.2015. Im Internet unter: https://vimeo.com/119433287 [20.08.2016].

Younes Benziane (2013): Egyptian Photographer Films His Own Death 08/07/2013. In: Youtube.com vom 10.08.2013. Im Internet unter: https:/www.youtube.co $\mathrm{m} /$ watch? $=18 \mathrm{~T} 80$ B3im_M [05.01.2019].

\section{Videospiele}

Doom (1993). id Software. MS-DOS/Sega 32X/Atari Jaguar/SNES/Windows. GT Interactive/cdv Software/Atari/Activision.

Doom 2 (1994). id Software. MS-DOS/Windows/Mac OS/Game Boy Advance/PC98/Tapwave Zodiac/PlayStation 3/Xbox 360. cdv Software Entertainment.

Sacred Defense: Protecting the Homeland And Holy Sites (2018). Dar Al-Manar. Windows 7/8/10. Dar Al-Manar.

Syrian Warfare (2017). Cats Who Play. Windows 7/8/10. Cats Who Play. Bury me, my Love (2017). Arte France/The Pixel Hunt/FIGS. iOS/Android. Playdius. Path Out (2017). Causa Creations. Windows Vista/7/8.1/10 \& Mac OS X. Causa Creations. 